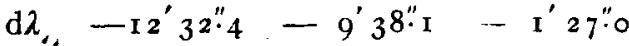

$$
\begin{aligned}
& \mathrm{d} \beta_{\prime \prime \prime}^{\prime \prime}-5^{6.6}-66^{6}-\circ 59.1
\end{aligned}
$$

Rechnet man in der bekannten Weise den Logarithmus der Cotangente des Winkels, den der durch die mittleren Cometen- und Sonnenorte gelegte grösste Kreis mit der Ekliptik einschliesst, so findet sich mit Benutzung

$\begin{array}{ccc}\text { der Beobachtungen } \log \operatorname{cotg} \mathcal{F} & 0.194795 \\ \text { mit dem ersten Elementensystem } & 0.194781 \\ \# \# \text { zweiten } & 0.19479^{2} \\ » \# \text { dritten } & & 0.194937\end{array}$

Es zeigt sich hieraus, dass durch eine Verbesserung in $M$ für die beiden ersten Lösungen kein wesentlich besserer Anschluss an die Beobachtungen erreicht werden kann, während sich $M$ für das dritte System verbesserungsfähig erweist; es ist demnach gar keinem Zweifel unterworfen, wie dies wohl schon anderweitig bekannt ist, dass die dritte Lösung die einzig brauchbare ist.

Wien 1882 October 29.

Th. v. Oppolzer.

\title{
Ueber die Kriterien des Vorhandenseins dreier Lösungen bei dem Cometenproblem.
}

In einer der kais. Akademie der Wissenschaften in Wien von mir überreichten Abhandlung führen sich die Kriterien für das Vorhandensein dreier Lösungen bei dem Cometenproblem auf die unten folgenden Formeln zurück.

Sind $h, g$ und $\cos \varphi$ die bekannten bei einer Cometenbahnbestimmung auftretenden Hilfsgrössen, $\lambda_{\mu}$ und $\beta_{u}$ die

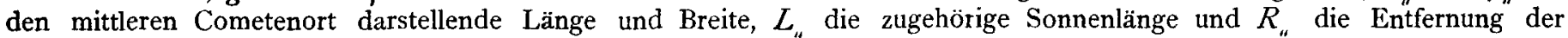
Erde von der Sonne, so ist'zunächst zu berechnen

und weiter:

$$
\alpha=\frac{h}{g} R_{u}, \cos \psi_{u}=\cos \beta_{n} \cos \left(\lambda_{n}-L_{u}\right)
$$

$$
\begin{aligned}
A_{1} & =-\frac{5}{3} \cos \psi_{\circ}-\frac{4}{3}\left(\frac{\cos \varphi}{\alpha}\right), & A_{2} & =\frac{2}{3}+2 \cos \psi_{1}\left(\frac{\cos \varphi}{\alpha}\right)+\frac{1}{3 \alpha^{2}} \\
A_{3} & =-\frac{2}{3}\left(\frac{\cos \varphi}{\alpha}\right)-\frac{\cos \psi_{1}}{3 \alpha^{2}}, & p & =\frac{\mathrm{I}}{3} A_{1}{ }^{2}-A_{2} \\
q & =\frac{2}{27} A_{1}{ }^{3}-\frac{\mathrm{I}}{3} A_{1} A_{2}+A_{3}, & r^{2} & =\frac{4}{3} p, \sin 3 \omega=\frac{4 q}{r^{3}},
\end{aligned}
$$

$p$ muss positiv und $4 q<r^{3}$ sein, wenn überhaupt drei brauchbare Lösungen vorhanden sein sollen; $r$ stets positiv und 300 im ersten Quadranten zu wählen und 300 erhält das Vorzeichen von $q$;

$$
x_{2}=r \sin \omega-\frac{\mathrm{I}}{3} A_{1}, x_{3}=r \sin \left(60^{\circ}-\infty\right)-\frac{\mathrm{I}}{3} A_{1}
$$

$x_{2}$ muss positiv sein, wenn überhaupt drei brauchbare Lösungen möglich sein sollen; dieselben sind in der That vorhanden, wenn den beiden Ungleichungen:

genügt .wird.

$$
\begin{aligned}
& \left(\alpha^{2} x_{2}{ }^{2}-2 \cos \varphi \cdot \alpha x_{2}+1\right)^{2}\left(x_{2}{ }^{2}-2 \cos \psi_{u} \cdot x_{2}+1\right)>4 R^{2}{ }^{2} \\
& \left(\alpha^{2} x_{3}{ }^{2}-2 \cos \varphi \cdot \alpha x_{3}+1\right)^{2}\left(x_{3}{ }^{2}-2 \cos \psi_{1} \cdot x_{3}+1\right)<4 \mathrm{R}{ }^{2}{ }^{2}
\end{aligned}
$$

Für das in der letzen Zeit am Cometen Cruls entdeckte Beispiel wird:

$$
\begin{aligned}
& \log \alpha=0.4427, \log \cos \psi_{u}=9.9974, \log \cos \varphi=9.7793 \mathrm{n}, \log \mathrm{R}_{u}=0.00 \mathrm{I} 7 \\
& A_{1}=-\mathrm{r.3674}, A_{2}=+0.2786, \quad A_{3}=+0.1016, \quad p=+0.3447 \\
& q=+0.0393, \log r=0.8312, \quad \omega=10^{\circ} 6^{\prime}, \quad x_{2}=+0.5747, x_{3}=+0.9743 \\
& \left(\alpha^{2} x_{2}^{2}-2 \cos \varphi \cdot \alpha x_{2}+1\right)^{2}\left(x_{2}{ }^{2}-2 \cos \psi_{11} \cdot x_{2}+1\right)=+5 \cdot 6 \\
& 4 R^{2}=+4.0 \\
& \left(\alpha^{2} x_{3}{ }^{2}-2 \cos \varphi \cdot \alpha x_{3}+1\right)^{2}\left(x_{3}{ }^{2}-2 \cos \psi_{10} \cdot x_{3}+1\right)=+ \text { I. }{ }^{4} \text {. }
\end{aligned}
$$

Wien 1882 November 3 .

Th. v. Oppolzer, 